\title{
Constraints on the disk geometry of the T Tauri star AA Tau from linear polarimetry ${ }^{\star}$
}

\author{
F. Ménard ${ }^{1}$, J. Bouvier ${ }^{1}$, C. Dougados ${ }^{1}$, S. Y. Mel'nikov ${ }^{2,3}$, and K. N. Grankin ${ }^{2,3}$ \\ ${ }^{1}$ Laboratoire d'Astrophysique de Grenoble, CNRS/UJF UMR 5571, 414 rue de la Piscine, BP 53, 38041 Grenoble Cedex 9 , \\ France \\ e-mail: jbouvier@obs.ujf-grenoble.fr; dougados@obs.ujf-grenoble.fr \\ 2 Astronomical Institute of the Academy of Sciences of Uzbekistan, Astronomicheskaya 33, Tashkent 700052, Uzbekistan \\ e-mail: kn@astrin.uzsci.net; smeln@sai.msu.ru \\ ${ }^{3}$ Isaac Newton Institute of Chile, Uzbekistan Branch, Santiago, Chile \\ e-mail: newton@reuna.cl
}

Received 27 March 2003 / Accepted 18 June 2003

\begin{abstract}
We have simultaneously monitored the photometric and polarimetric variations of the Classical T Tauri star AA Tau during the fall of 2002. We combine these data with previously published polarimetric data covering two earlier epochs. The phase coverage is complete, although not contiguous. AA Tau clearly shows cyclic variations coupled with the rotation of the system. The star-disk system produces a repeatable polarisation curve where the polarisation increases with decreasing brightness. The data fit well with the model put forward by Bouvier et al. (1999) where AA Tau is viewed almost edge-on and its disk is actively dumping material onto the central star via magnetospheric accretion. The inner edge of the disk is deformed by its interaction with the tilted magnetosphere, producing "eclipses" as it rotates and occults the photosphere periodically. From the shape of the polarisation curve in the $Q U$-plane we confirm that the accretion disk is seen at a large inclination, almost edge-on, and predict that its position angle is PA $\sim 90^{\circ}$, i.e., that the disk's major axis is oriented in the East-West direction.
\end{abstract}

Key words. accretion: accretion disks - stars: circumstellar matter - stars: pre-main sequence - polarization stars: individual: AA Tau

\section{Introduction}

Classical T Tauri stars (CTTS) are low-mass pre-main sequence stars surrounded by accretion disks. The current models for these star-disk systems propose that the disks are truncated at a few stellar radii by a powerful stellar magnetic field. Accretion onto the central star is proceeding along the field lines, from the inner edge of the disk to the star, at near free-fall velocities. It is the so-called magnetospheric accretion model. Observational evidence for this scenario has been growing steadily, e.g., Bertout et al. (1988), Edwards et al. (1994), Alencar et al. (2001).

One of the stringent assumptions of this model is the topology and strength of the stellar magnetic field required. It is usually assumed to be dipolar with a strength of the order of a few kiloGauss or so. Apart from a few observations (e.g., Guenther et al. 1999; Johns-Krull et al. 1999, 2001; Donati et al. 2000),

Send offprint requests to: F. Ménard,

e-mail: menard@obs.ujf-grenoble.fr

* Based on observations collected with the STERENN Polarimeter at the $2 \mathrm{~m}$ Bernard-Lyot telescope (TBL) operated by INSU/CNRS and Pic-du-Midi Observatory (CNRS USR 5026), France. its exact nature remains poorly constrained. A few authors have argued that the field may not be aligned with the stellar rotation axis in a few cases (e.g., Kenyon et al. 1994 for DR Tau; Johns \& Basri 1995 for SU Aur; Bouvier et al. 1999 for AA Tau).

To probe further into the details of magnetospheric accretion, we have obtained spectro-photometric synoptic observations of a few representative CTTS, including AA Tau (Bouvier et al. 1999, hereafter B99). Continuous coverage over several rotation periods provided a detailed view of the star-disk interaction, hence of the physics of the accretion process itself.

Specific to AA Tau, B99 proposed a model where the stardisk system is seen almost edge-on and where the inner edge of the disk is deformed by its interaction with the magnetic field. As a consequence and as the disk rotates, occulting material moves in and out of the line-of-sight, occulting the star and producing quasi-cyclic fadings. Because the dimming episodes are not strictly identical, even from one cycle to the next, the accretion process is shown to be non-steady, with the massloading of the field lines and the deformation of the disk highly time-dependent (Bouvier et al. 2003).

All these phenomena occur well beyond the resolution limit of current telescopes and one will have to wait for the next 
Table 1. Linear polarisation measurements of AA Tau.

\begin{tabular}{|c|c|c|c|c|c|}
\hline JD & filter & $\begin{array}{l}P \\
(\%)\end{array}$ & $\begin{array}{l}\sigma(P) \\
(\%)\end{array}$ & $\begin{array}{l}\theta \\
\left({ }^{\circ}\right)\end{array}$ & $\begin{array}{l}\sigma(\theta) \\
\left({ }^{\circ}\right)\end{array}$ \\
\hline \multicolumn{6}{|c|}{ Data set $1: 7-18 / 10 / 1984$} \\
\hline 2445980.952 & 4700 & 0.73 & 0.07 & 43.3 & 2.5 \\
\hline 2445982.862 & 4700 & 2.14 & 0.11 & 12.6 & 1.5 \\
\hline 2445983.914 & 4700 & 2.35 & 0.21 & 11.3 & 2.5 \\
\hline 2445991.801 & 4700 & 1.68 & 0.23 & 12.0 & 3.9 \\
\hline 2445991.840 & 4700 & 1.75 & 0.22 & 6.4 & 3.5 \\
\hline \multicolumn{6}{|c|}{ Data set 2: $23-29 / 11 / 1995$} \\
\hline 2450042.823 & V & 0.72 & 0.01 & 25.0 & 1.0 \\
\hline 2450043.979 & V & 0.70 & 0.01 & 24.5 & 1.0 \\
\hline 2450044.891 & $V$ & 1.08 & 0.01 & 14.8 & 1.0 \\
\hline 2450048.547 & V & 1.33 & 0.13 & 2.2 & 2.4 \\
\hline 2450049.525 & $V$ & 0.60 & 0.09 & 20.3 & 3.7 \\
\hline 2450049.676 & V & 0.80 & 0.21 & 20.8 & 6.5 \\
\hline 2450050.638 & V & 0.84 & 0.10 & 27.5 & 2.9 \\
\hline \multicolumn{6}{|c|}{ Data set 3: $30 / 10-5 / 11 / 2002$} \\
\hline 2452577.586 & $I$ & 0.80 & 0.05 & 27.8 & 2.0 \\
\hline 2452579.520 & $I$ & 2.11 & 0.06 & 7.0 & 1.1 \\
\hline 2452580.544 & $I$ & 0.54 & 0.06 & 37.0 & 3.1 \\
\hline 2452583.510 & $I$ & 0.47 & 0.03 & 34.0 & 2.0 \\
\hline 2452584.651 & $I$ & 0.64 & 0.04 & 19.0 & 1.9 \\
\hline
\end{tabular}

generation of long-baseline optical/near-infrared interferometers for any hope of directly imaging these regions. Until then, "tomography" as obtained by synoptic campaigns is our best tool to understand the details of the linkage between the stellar magnetosphere and its accretion disk and therefore to understand the fundamental mass-accretion process by which solarlike stars form.

In the following sections we present photometric and linear polarimetric monitoring of AA Tau in an attempt to solve some of the issues raised by the model proposed by B99. First, is it really circumstellar extinction that causes the quasi-periodic fadings? Second, what is the 3-dimensional orientation of the disk, is it really edge-on?

Understanding these questions goes beyond understanding AA Tau itself. It will help demonstrate whether or not the accretion topology derived for AA Tau can be applied more generally to other CTTS.

\section{Observations and data reduction}

\subsection{Linear polarimetry}

Three data sets are presented in Table 1 . Data sets $1 \& 2$ were published previously by Ménard \& Bastien (1992) and B99, respectively. Data set 3 is new. It was obtained with the STERENN polarimeter attached to the $2 \mathrm{~m}$ Bernard-Lyot Telescope of the Pic-du-Midi Observatory, France. The observations were performed during the period 30 October-5 November $2002^{1}$.

\footnotetext{
1 The same instrument was used to collect some of the data in set 2, but the detectors were different.
}

The data reduction procedure for data sets $1 \& 2$ is presented in the original papers. In the fall of 2002, for data set 3 , the efficiency and stability of the instrument was checked every night by measuring at least 2 of a set of 4 highly polarised standard stars. Night-to-night efficiency corrections were applied to account for small variations in the instrument efficiency. The average efficiency of the instrument was 0.95 . A constant shift of $+1.25^{\circ}$ in position angle (i.e., obs. value $+1.25=$ true value), induced by the chromaticity of the rotating half-wave plate was carefully measured and removed from the data. We found no instrumental polarisation by measuring well known unpolarised standard stars and numerous nearby M-dwarfs during moonfree time. With a typical $1-\sigma$ error of $\sigma(P)=0.02 \%$, all these measurements are compatible with zero polarisation and no correction was applied to the data.

In Table 1, the julian date of the middle of the observation is given for each datum. The next column gives the central wavelength of the filter or the photometric band used. Then follow the polarisation, $P$, its associated error, $\sigma(P)$, the polarisation position angle given in the equatorial system, $\theta$, and its associated error, $\sigma(\theta)$. The error $\sigma(\theta)$ was increased to $1.0^{\circ}$ systematically in cases where it would have been smaller formally.

\subsection{Optical photometry}

The optical aperture photometry used here was obtained at the $60 \mathrm{~cm}$ telescope of the Mount Maidanak Observatory, Uzbekistan, during the period 13 September1 December 2002. A photometer with a pulse counting FEU-79 photomultiplier tube was used with standard $U B V R$ Johnson filters and a $15^{\prime \prime}$ diaphragm. Typical exposure times ranged from 50 up to $120 \mathrm{~s}$, depending on the filter and on the brightness of the star. During one of the photometric nights of the run, secondary standards were observed for flux calibration. The data were reduced with the standard procedures using the mean extinction coefficients for the observatory. The final observing error is $\sim 0.01 \mathrm{mag}$. on average. In this paper we present only the $V$-band data overlapping with the polarisation observations. All other data will be made available elsewhere.

AA Tau's photometry has also been monitored prior to this work and rotational periods of roughly 8.2 days have been reported, e.g., Vrba et al. (1989) and Herbst et al. (1994). B99 presented the results of a large synoptic campaign performed in 1995, matching with data set 2 of Table 1. In that campaign, the $8.2 \mathrm{~d}$ rotational period was recovered and quasicyclic episodes of fadings were found due to the improved time coverage, see also Fig. 2. The behaviour of AA Tau in the fall of 2002 , i.e., in the data presented here, appears very similar, see Fig. 3.

\section{Results}

\subsection{Linear polarimetry}

Although the data presented in Sect. 2.1 were obtained in 3 different filters, they clearly show, globally but also within each set, that the linear polarisation of AA Tau is variable. This confirms the variability analysis of Ménard \& Bastien (1992). 
The maximum polarisation observed is $P_{\max }=2.35 \%$ in the blue and $P_{\max }=2.1 \%$ in the red. Both values are similar, within $1-\sigma$. The minimum value observed is $P_{\min }=0.6 \%$ in the blue and $P_{\min } \sim 0.5 \%$ in the red, also similar within 2- $\sigma$. The data is plotted in Fig. 1 where the normalised Stokes parameters $Q$ and $U$ are plotted. They are related to the usual polarisation, $P$, and position angle, $\theta$, by the following relations:

$Q=P \cos (2 \theta), U=P \sin (2 \theta)$.

Clearly, the data are not distributed randomly in the $Q U$-plane. Each measurement lies on an elongated strip. This strip is bounded by $0.0 \% \leq Q \leq 2.2 \%$ and $0.1 \% \leq U \leq 0.9 \%$. It is elongated mostly in along the $Q$-axis. It is important to note that the three data sets, taken in three different filters at three different epochs, blend in smoothly within the same strip. In Sect. 4 we will argue that this behaviour of the polarisation in the $Q U$-plane is intimately linked to the geometry of the circumstellar environment of AA Tau.

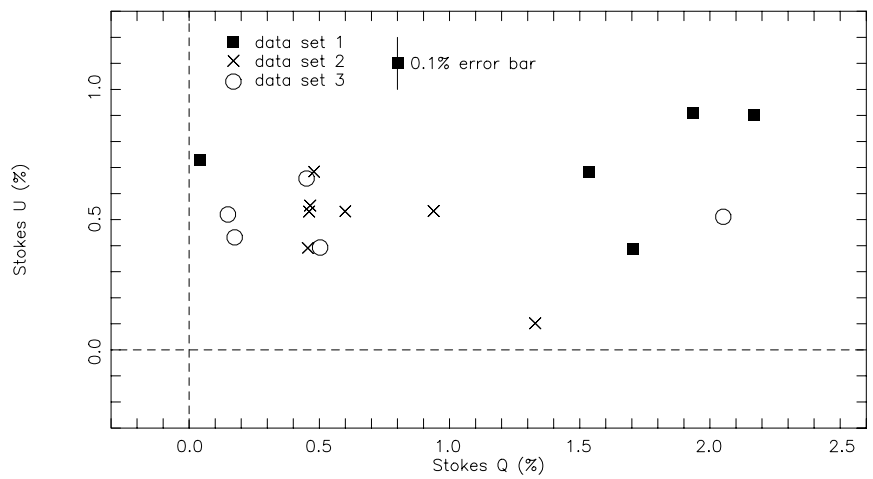

Fig. 1. Plot of the Stokes parameters observed for AA Tau. The filled squares show data set 1 , the crosses show data set 2 , and the empty circles show data set 3 . The dashed-lines trace the lines where $Q$ and $U$ are equal to zero, for reference. A representative $0.1 \%$ error bar is shown for comparison.

\section{Discussion}

B99 presented an analysis of the variability of AA Tau based on extensive multi-site continuous spectro-photometric monitoring of the star. From the light curve, they find a rotation period of 8.2 days. The light curve is quite unusual for a T Tauri star. It is roughly constant, in the bright state, with quasi-periodic fadings of amplitude up to $1.4 \mathrm{mag}$ in BVRI. Because of the lack of color variations during the fadings, B99 interpreted the data in terms of occultations of AA Tau's photosphere by opaque circumstellar material.

To produce the observed occultations, they proposed that the inner edge of the accretion disk is bent, warped by a large scale dipole tilted with respect to the stellar rotation axis and causing a "piling-up" of material preferentially on one side. This piling-up results in non-axisymmetric vertical distribution of dust, the occulting material. As the inner edge of the disk rotates, in corotation with the star, it eclipses the photosphere partially every 8.2 days.

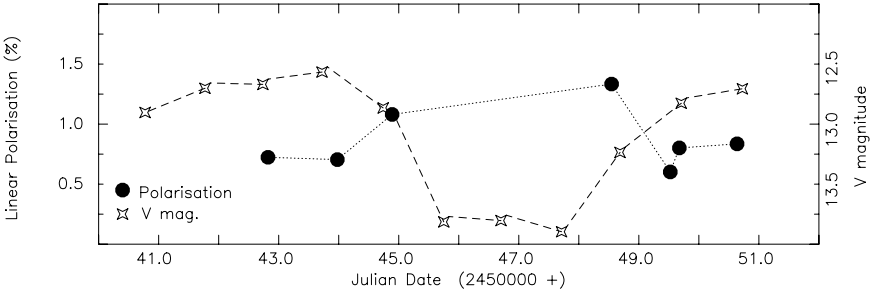

Fig. 2. Plot of the polarimetric and photometric variations of AA Tau as a function of time during part of the 1995 campaign. Data are from B99. Full circles \& dotted line: $V$-band linear polarisation of AA Tau as a function of Julian date. Open stars \& dashed line: $V$-band photometry as a function of Julian date.

This magnetospheric accretion model has the ability to explain the optical and near-infrared light curves and the veiling in a coherent way. However, albeit interesting, the model raises a number of issues that one must solve in order to push it further. This section will address two of these issues. First, are the fadings seen in the light curve really caused by extinction? Second, is the accretion disk tilted to a large inclination, next to edge-on, as needed to produce eclipses?

Answering these questions is important to confirm the magnetospheric accretion model for AA Tau and understand the origin of the peculiarities of its light curve and therefore study the applicability of AA Tau's model to other T Tauri stars.

\subsection{Evidence for circumstellar extinction}

AA Tau's light variations have roughly neutral colors during the fadings, sometime exhibiting a slight blueing at minimum light (B99). This is very reminiscent of UXORs where deep minima are found, with blueing for the deepest ones. The case for circumstellar extinction is well documented for UXORs (Natta et al. 2000, and references therein). Interestingly, the polarisation of UXORs increases when the stars fade. This is because the relative fraction of scattered (hence polarised) light increases as the photosphere becomes occulted by an optically thick clump of circumstellar material.

B99 presented sparse linear polarimetry with their photometry. The polarisation shows a hint of increasing when AA Tau fades but the measurements did not cover a full occultation, see Fig. 2 where we reproduce the relevant data. Therefore, no firm conclusions could be reached.

In Fig. 3 we plot the polarimetric data and the $V$-band photometry of data set 3 (see Table 1 ) as a function of time. Clearly, close to JD $=2452579.5$ the polarisation rose from the minimum level $\left(P_{\min }\right)$ to the maximum value $\left(P_{\max }\right)$, and decreased back to the minimum level as the light curve went through a minimum. The maximum polarisation is coincident with the minimum brightness of AA Tau. This is a strong indication that extinction is responsible for the fading of AA Tau and scattering in the nearby circumstellar environment responsible for the polarisation. 


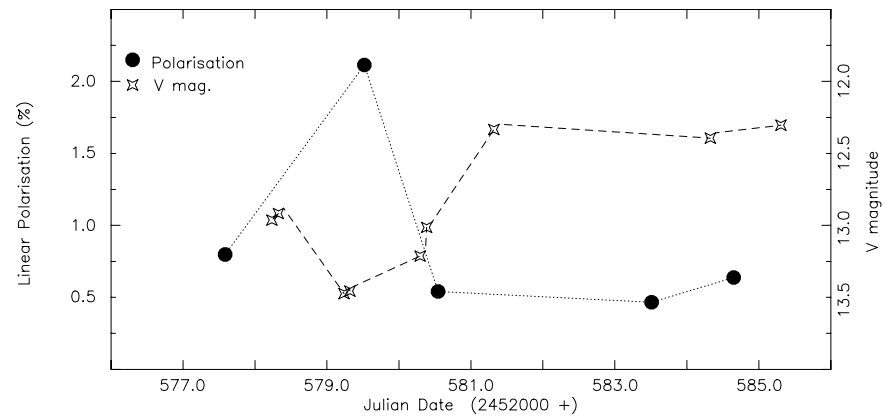

Fig. 3. Plot of the polarimetric and photometric variations of AA Tau as a function of time. Full circles \& dotted line: $I$-band linear polarisation of AA Tau as a function of Julian date. Open stars \& dashed line: $V$-band photometry as a function of Julian date.

\subsection{Geometry of the occulting material}

In the model envisioned by B99, the maximum height of the warped disk occurs at the same longitude as the photospheric hot spot, which is also the longitude of the tilted magnetic pole. This is probably an over simplification and further monitoring and numerical simulations will help study this question in greater details. For now, we will consider that the disk is deformed and its increased vertical extension is causing the observed occultations. Irrespective of the exact azimuthal location of this vertical extent though, simple geometric arguments are sufficient to deduce the inclination to the line-of-sight of the accretion disk from the temporal variations of the polarisation curve, i.e., its shape in the $Q U$-plane (see Fig. 1).

For a perfectly axisymmetric star-disk system, the ratio of direct unpolarised light to scattered polarised light remains constant with rotational phase. Photometry and polarimetry are constant in that case. Including an asymmetry, e.g., a warped inner edge in the disk, modifies the amount of scattered flux received by an observer in two ways compared to the axisymmetric case. First, the total brightness of the star-disk system will not remain the same. Second, it will also be modulated as the system rotates.

For an observer viewing the system equator-on, the light distribution will go through maximum amplitude variations as the warp in the disk moves in and out of the line-of-sight, i.e., occulting the photospheric disk in a variable way. The polarisation position angle will remain constant because the disk is projected as a straight line in the plane of the sky. In this case, only the total brightness and polarisation level will change as the asymmetry moves in and out of the line-of-sight.

On the contrary, an observer viewing the same nonaxisymmetric system pole-on would not detect a rotational modulation in the photometry because the whole system is always viewed with the same aspect. Only a rotation in the distribution of scattered light (i.e., in the brightness map) and a rotation in the polarisation position angle would occur, but without amplitude modulations.

For AA Tau, as shown in Fig. 1, all the data points fall within a narrow elongated strip, i.e., the polarisation level is modulated but its position angle is constant. This argues strongly in favor of a star-disk system seen at a large inclination, almost edge-on. The non-zero width of the strip where the data fall in Fig. 1 can have several origins. On the one hand, the system may not be perfectly edge-on and the polarisation curve not a perfect straight line. On the other hand, the scattering geometry is also likely to be more complex than assumed here. Finally, long term variability and non-zero error bars may contribute to increase the observed width.

\subsection{Orientation of the accretion disk}

The arguments presented in Sect. 4.2 can also be used to predict the position angle of the disk, the other angle defining its complete orientation in 3D space. Indeed, we deduced that the inclination is large. For simplicity, we will now assume that the system is exactly edge-on and the polarisation curve in the $Q U$-plane (see Fig. 1) is a straight line that is running left-right. We will also assume that scattering on dust in responsible for the polarisation. This is reasonable in comparison with other accretion disks observed in $\mathrm{T}$ Tauri stars by scattered light in the optical and near-infrared (e.g., Ménard \& Bertout 1999 for a review). In that case, the net polarisation is usually perpendicular to the plane of the disk in the absence of a large envelope (e.g., Whitney \& Hartmann 1992). In Fig. 1, the polarisation curve runs parallel to the $Q$-axis. In the equatorial coordinate system this is along the North-South direction, i.e., at a position angle $\sim 0^{\circ}$. If the polarisation is indeed perpendicular the plane of the disk, then we predict that AA Tau's light scattering disk should be oriented more or less along the East-West direction.

\subsubsection{On the variations of the observed position angle}

In general the Stokes parameters are the algebraic sum of two (or more) contributions, intrinsic and interstellar:

$Q_{\text {observed }}=Q_{\text {intrinsic }}+Q_{\mathrm{ISM}}$,

$U_{\text {observed }}=U_{\text {intrinsic }}+U_{\text {ISM }}$.

The observed polarisation position angle is defined as $\theta=$ $\frac{1}{2} \operatorname{arctg}\left(\frac{U_{\text {obs }}}{Q_{\text {obs }}}\right)$.

In the previous section we used the fact that the polarisation curve is oriented along the $Q$-axis to predict that the disk will be oriented East-West. In reality the position angles we measure are variable and in the range $2^{\circ} \leq \theta \leq 43^{\circ}$ instead, not quite at $0^{\circ}$. We show here that this behaviour is not intrinsic to the source.

In general, the interstellar polarisation is assumed to be constant. From Eqs. (2), it is immediate to see that a constant $Q_{\text {ISM }}$ and $U_{\text {ISM }}$ induce only a shift of the intrinsic polarisation curve in the $Q U$-plane. Any favored behaviour in the polarisation curve will therefore trace the intrinsic properties of the system. We need not care about the shift from zero of AA Tau's measured polarisation curve in the $Q U$-plane to estimate the orientation of its circumstellar environment. What matters is the general orientation of this curve in Fig. 1 .

To go further, we can show that $U_{\text {intrinsic }} \simeq 0$, hence that $\theta_{\text {intrinsic }} \simeq 0$ for $\mathrm{AA}$ Tau. The observed variations in position angles being due only to $U_{\text {observed }} \neq 0$ because $U_{\text {ISM }} \neq 0$ and $Q_{\text {obs }}$ being variable. 
To verify that the shift towards the $+U$ axis of AA Tau's polarisation curve is caused only by interstellar polarisation, i.e., $U_{\text {observed }}=U_{\text {ISM }}$, we compare AA Tau's polarisation with that of other nearby T Tauri stars. Within $30 \mathrm{arcmin}$ of AA Tau, DN Tau $\left(P=0.6 \%, \theta=34^{\circ}\right)$, GI Tau $\left(P=0.8 \%, \theta=65^{\circ}\right)$, GK Tau $\left(P=1.25 \%, \theta=42^{\circ}\right)$, and V830 Tau $(P=0.46 \%$, $\theta=53^{\circ}$ ) are found for which we have polarisation measurements in the $I$-band, or similar. Of these, V830 Tau is a weakline $\mathrm{T}$ Tauri star. It is not expected to have a significant amount of circumstellar dust, hence it should not show a large intrinsic polarisation. We therefore attribute an interstellar origin to its observed polarisation. Because it is located near AA Tau, it is reasonable to assume that AA Tau also has an interstellar polarisation of the same order, i.e., $P \sim 0.5 \%$ at $\sim 50^{\circ}$. According to Eq. (1), this polarisation has the following Stokes parameters: $Q_{\text {ISM }}=-0.09 \%, U_{\text {ISM }}=+0.49 . \%$.

The shift of AA Tau's measurements along the $Q$-axis caused by interstellar polarisation is minimal because $Q_{\text {ISM }}$ is small, as measured on V830 Tau. However, the observed shift along the $U$-axis is of order $0.5 \%$ and can be attributed entirely to the interstellar component since $U_{\text {ISM }} \sim+0.5 \%$. Removing the interstellar contribution brings AA Tau's curve down almost exactly on the $Q$-axis.

As a consequence, we believe that the variations of the observed linear polarisation position angle are not intrinsic to AA Tau and do not reflect a rapid change in the geometry of its circumstellar environment. We predict that the light scattering disk is oriented in the East-West direction.

\subsection{Comparison with other studies}

Dutrey et al. (1996) observed the dust thermal emission of AA Tau at $2.7 \mathrm{~mm}$ with the IRAM interferometer. A flux of $13.2 \pm 1.8 \mathrm{mJy}$ was measured but the source remained unresolved in the $\sim 3^{\prime \prime}$ beam. Recently, Kitamura et al. (2002) re-observed AA Tau with the Nobeyama millimeter array. At $2 \mathrm{~mm}$, they measured a flux of $22 \pm 3$ and $13 \pm 2 \mathrm{mJy}$ in compact ( $5^{\prime \prime}$ beam) and extended ( 1 " beam) configurations, respectively. AA Tau is marginally resolved in their images. They find a major axis of $1.34 \pm 0.1$ arcsec and a minor axis of $0.61 \pm$ $0.13 \mathrm{arcsec}$ (with a synthesized beam size of 1.4 and $1.3 \mathrm{arcsec}$ along the major and minor axis respectively).

The major axis of the outer disk is well resolved and Kitamura et al. (2002) estimate a reliable position angle of $86^{\circ} \pm 5^{\circ}$. This is in very good agreement with our suggestion that the inner disk runs East-West (see Sect. 4.3).

However, the minor axis of AA Tau's disk is only marginally resolved at radio wavelengths and the inclination, calculated as the ratio of minor to major axis, is probably overestimated by their measurements. They ran two different models to fit their data and obtained inclination values in the range $i=25-28^{\circ}$, not compatible with the model of B99 nor with the estimations presented in Sect. 4.2. Finer millimeter observations with more extended arrays and at higher frequencies are needed to check these results and solve this discrepancy.

\section{Conclusions}

We presented optical linear polarisation data of the Classical T Tauri star AA Tau. The data were obtained at three different epochs and show a remarkably stable behaviour: a low-level polarisation when the star+disk system is bright and a significant increase in polarisation when the system dims.

These results allow us to confirm that extinction by circumstellar material is responsible for the quasi-periodic fading episodes observed in the light curve. Furthermore, the distinctive shape of the polarisation curve strongly supports the idea that the occulting material orbiting AA Tau is located in a disk viewed at a large inclination, close to edge-on. Finally, the disk's major axis is likely oriented in the East-West direction.

These results allow to confirm and refine the magnetospheric accretion model put forward for AA Tau. The orientation of AA Tau's disk provides the solution as to why its light curve is different from that of most other classical T Tauri stars: the "eclipses" are a direct consequence of its large tilt to the line-of-sight.

Acknowledgements. The authors gratefully acknowledge financial support from Université Joseph Fourier, Grenoble (1994 BQR grant B 644 R1), the Laboratoire d'Astrophysique de Grenoble, and the Observatoire Midi-Pyrénées for upgrade and maintenance of the STERENN polarimeter. Financial support for the observations at Pic-du-Midi was provided by the Programme National de Physique Stellaire (PNPS) of CNRS/INSU, France.

\section{References}

Alencar, S. H. P., Johns-Krull, C. M., \& Basri, G. 2001, AJ, 122, 3335 Bertout, C., Basri, G., \& Bouvier, J. 1988, ApJ, 330, 350

Bouvier, J., Chelli, A., Allain, S., et al. 1999, A\&A, 349, 619 (B99)

Bouvier, J., Grankin, K. N., Alencar, S., et al. 2003, A\&A, 409, 169

Donati, J.-F., Mengel, M., Carter, B. D., et al. 2000, MNRAS, 316, 699

Dutrey, A., Guilloteau, S., Duvert, G., et al. 1996, A\&A, 309, 493

Edwards, S., Hartigan, P., Ghandour, L., \& Andrulis, C. 1994, AJ, 108, 1056

Guenther, E. W., Lehmann, H., Emerson, J. P., \& Staude, J. 1999, A\&A, 341, 768

Herbst, W., Herbst, D. K., Grossman, E. J., \& Weinstein, D. 1994, AJ, 108, 1906

Johns, C. M., \& Basri, G. 1995, ApJ, 449, 341

Johns-Krull, C. M., Valenti, J. A., Saar, S. H., \& Hatzes, A. P. 2001, in Cool Stars, Stellar Systems, and the Sun, ed. R. J. Garcia López, R. Rebolo, \& M. R. Zapatero Osorio (San Francisco: ASP), ASP Conf. Ser., 223, 521

Johns-Krull, C., Valenti, J. A., Hatzes, A. P., \& Kanaan, A. 1999, ApJ, 501, L41

Kenyon, S. J., Hartmann, L., Hewett, R., et al. 1994, AJ, 107, 2153

Kitamura, Y., Momose, M., Yokogawa, S., et al. 2002, ApJ, 581, 357

Ménard, F., \& Bastien, P. 1992, AJ, 103, 564

Ménard, F., \& Bertout, C. 1999 in The Origin of Stars and Planetary Systems, ed. C. J. Lada, \& N. D. Kylafis (Dordrecht: Kluwer), 341 Natta, A., Grinin, V. P., \& Mannings, V. 2000, in Protostars and Planets IV, ed. V. Mannings, A. P. Boss, \& S. S. Russell (Tucson: University of Arizona Press), 559

Vrba, F. J., Rydgren, A. E., Chugainov, P. F., Shakovskaia, N. I., \& Weaver, W. B. 1989, AJ, 97, 483

Whitney, B. A., \& Hartmann, L. 1992, ApJ, 395, 529 